\title{
Rare condition of needle tract seeding after EUS-guided FNA for intraductal papillary mucinous carcinoma
}

Authors

Institutions
Akane Yamabe $^{1}$, Atsushi Irisawa ${ }^{1}$, Goro Shibukawa ${ }^{1}$, Koki Hoshi ${ }^{1}$, Mariko Fujisawa', Ryo Igarashi ${ }^{1}$, Ai Sato ${ }^{1}$, Takumi Maki ${ }^{1}$, Hiroshi Hojo ${ }^{2}$

${ }^{1}$ Department of Gastroenterology. Fukushima Medical University Aizu Medical Center, Aizuwakamatsu, Japan

${ }^{2}$ Department of Pathology, Fukushima Medical University Aizu Medical Center, Aizuwakamatsu, Japan submitted

1. March 2016

accepted after revision

18. April 2016

\section{Bibliography}

Dol http://dx.doi.org/

10.1055/s-0042-107072

Published online: 19.5.2016

Endoscopy International Open

2016; 04: E756-E758

(c) Georg Thieme Verlag KG

Stuttgart · New York

E-ISSN 2196-9736

\section{Corresponding author}

\section{Atsushi Irisawa, MD, PhD}

Department of

Gastroenterology

Fukushima Medical University

Aizu Medical Center

21-2, Maeda, Tanisawa,

Kawahigashi

Aizuwakamatsu 969-3492

Japan

irisawa@fmu.ac.jp

\section{License terms}

(ब) (1) $\ominus$
Background and study aims: We report on a 75year-old man who was admitted due to pancreatic cystic lesion accompanied by a solid mass with liver metastasis. Endoscopic ultrasound-guided fine-needle aspiration (EUS-FNA) was performed on the solid mass, and pathological findings revealed the lesion to be an adenocarcinoma diagnosed as intraductal papillary mucinous carcino-

\section{Introduction}

\section{$\nabla$}

Attempts to characterize pancreatic cysts include noninvasive endoscopic ultrasound (EUS) imaging. Although targeted endoscopic ultrasoundguided fine-needle aspiration (EUS-FNA) of the cyst wall following fluid aspiration has demonstrated improved overall diagnostic sensitivity for potentially malignant mucinous cysts [1], there are reports concerning a dissemination risk from EUS-FNA. The enhanced diagnostic capability of EUS-FNA must be balanced against the risk of tumor seeding.

\section{Case Report \\ $\nabla$}

A 75-year-old man was admitted to our hospital due to a pancreatic cystic lesion accompanying a solid mass, and with liver metastasis. EUS observation (UC240P-AL5, Olympus Co, Tokyo, Japan) demonstrated a pancreatic cyst of 30-mm maximum diameter, and a solid mass beside the cystic lesion. Using a 25-gauge needle (Expect, Boston Scientific Japan, Tokyo) with a $20-\mathrm{mL}$ syringe suction, we performed EUS-FNA on the solid mass, which also had a 30-mm maximum diameter through the posterior gastric wall. During the procedure, no early adverse events occurred. The pathological findings showed the lesion to be an adenocarcinoma that was diagnosed as intraductal papillary mucinous carcinoma (IPMC) (๑ Fig. 1). ma (IPMC). Approximately 3 months after, a cystic subepithelial lesion appeared in the posterior gastric wall where the EUS-FNA had been performed. We performed EUS-FNA again, which revealed that the cystic mass was IPMC with pathology similar to the original lesion. This is a rare case demonstrating needle tract seeding of EUSFNA for IPMC.

Because the patient's condition was inoperable, gemcitabine-based chemotherapy was started at a dose of $1600 \mathrm{mg}$ on days 1,8 , and 15 on a 4week cycle. Approximately 3 months after EUSFNA, follow-up computed tomography scan showed a cystic mass in the posterior gastric wall ( $\bullet$ Fig. 2). The cystic mass was visualized by esophagogastroduodenoscopy (EGD) as a subepithelial lesion (SEL) ( $\bullet$ Fig. 3), and EUS suggested that this SEL was primarily localized in the third layer of the gastric wall. Needle tract seeding of EUS-FNA for intraductal papillary mucinous carcinoma (IPMC) was suspected as the cause of this new lesion. EUS-FNA was thus performed on the SEL and a definite diagnosis of IPMC that was similar to previous pathological findings was obtained ( $\bullet$ Fig. 4). Because the cystic mass was located where the EUS-FNA had been performed, the SEL was therefore compatible with needle tract seeding from the initial EUS-FNA. As a second-line treatment, the patient underwent combined chemotherapy with one course comprising intravenous infusion of gemcitabine $1600 \mathrm{mg}$ on Day 1 and S-1 (tegafur + gimeracil+oteracil) $500 \mathrm{mg}$ twice a day for 14 days with a 1 -week rest period in between courses. Despite chemotherapy, he died from the cancer 29 months after the initial EUS-FNA. 


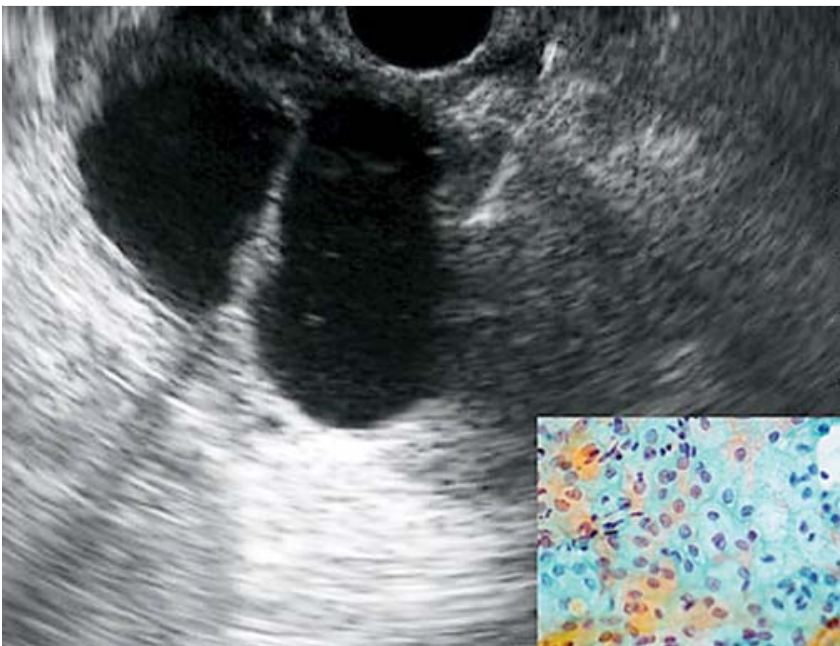

Fig. 1 EUS-FNA on a mass beside cystic lesions through the posterior gastric wall using a 25-gauge needle (Expect, Boston Scientific Japan, Tokyo). EUS-FNA for the pancreatic mass revealed differentiated tubular adenocarcinoma.

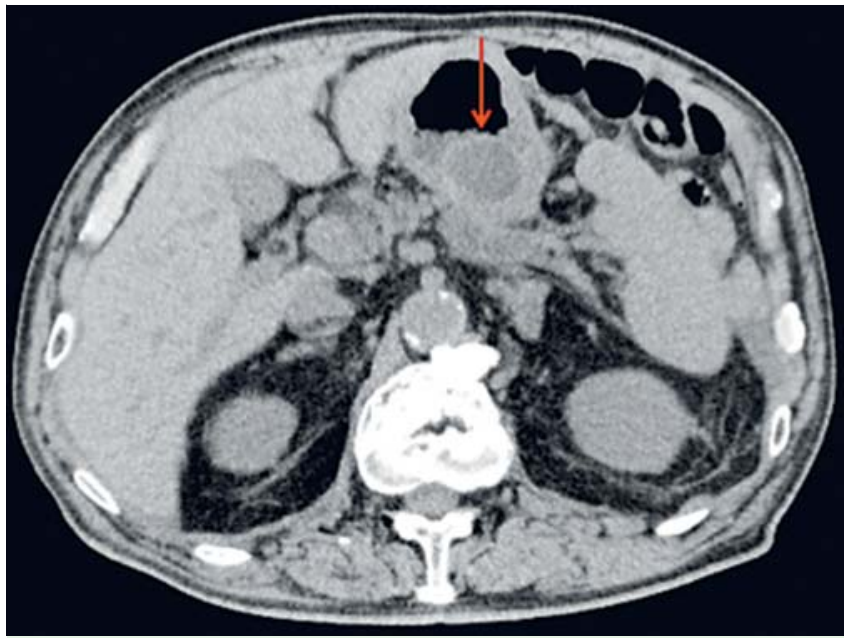

Fig. 2 A follow-up CT scan 3 months after EUS-FNA showed a new cystic lesion in the posterior gastric wall (arrow).

\section{Discussion}

\section{$\nabla$}

EUS-FNA for pancreatic cystic lesions is increasingly being used to differentiate benign cysts from cysts that have malignant potential or those with frank malignancy that may have otherwise been misdiagnosed as benign [2,3]. Pais et al. [3] reported that the sensitivity, specificity and accuracy of EUS-FNA for the diagnosis of malignancy in IPMC were 75\%, $91 \%$, and $86 \%$, respectively. Although a retrospective study reported no associated increased rate of gastric or peritoneal cancer recurrence following EUS-FNA [4], Levy et al. [5] demonstrated the potential for tumor cell displacement during EUS-FNA. In the study by Levy et al., the authors assessed the prevalence of luminal fluid-positive cytology among patients with luminal, extraluminal, and benign disease, and positive cytology was detected within post EUS-FNA luminal fluid in 3 of 26 patients (11.5\%) with pancreatic cancer. Cytological examination of luminal fluid aspirates did not demonstrate malignant cells in patients with nonmalignant disease, suggesting that the process of EUS-FNA may withdraw malignant

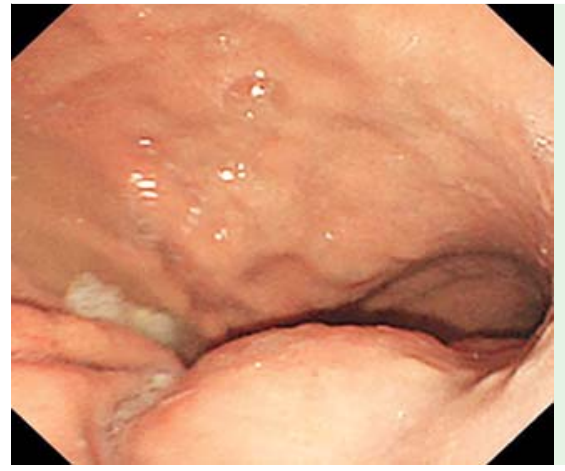

Fig. 3 EGD showing a subepithelial lesion-like mass on the posterior gastric wall.

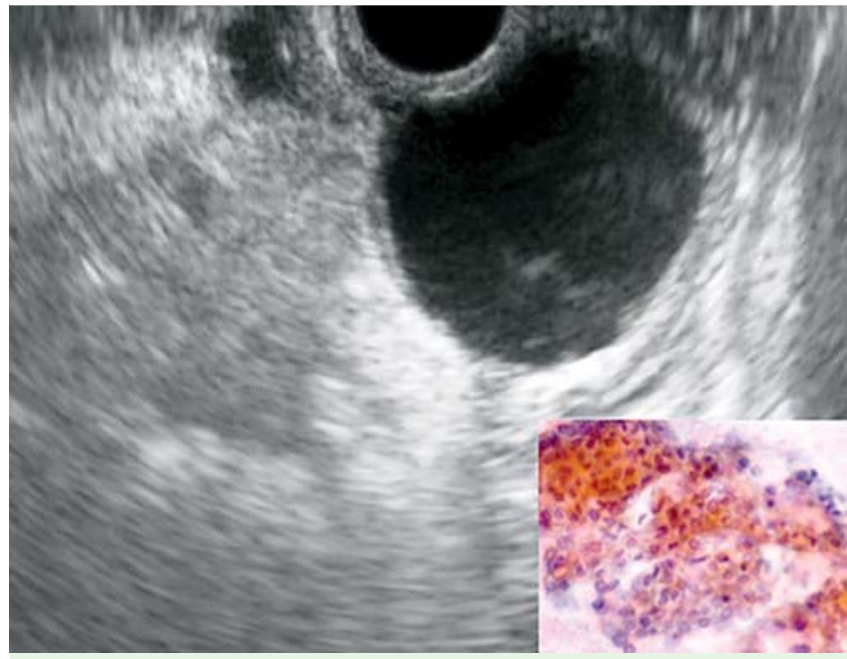

Fig. 4 EUS showed an echoic cystic mass measuring $24 \mathrm{~mm}$ in diameter, primarily located in the third layer of the gastric wall. EUS-FNA for the gastric cystic lesion revealed a differentiated tubular adenocarcinoma that was consistent with the primary tumor in the pancreas.

cells from a pancreatic cancer into the gastrointestinal luminal tract. This is likely an analogous method by which needle tract seeding occurs, and is concordant with reports on a dissemination risk from EUS-FNA. With respect to IPMC, Hirooka et al. [6] reported a case of IPMC dissemination to the peritoneum that was caused by EUS-FNA. Due to copious mucus production in IPMC, mucus production after needle tract seeding may lead to widespread dissemination. In cases where EUS-FNA is performed for a cystic solid mass, it is necessary to consider the possibility of needle tract seeding of IPMC. Therefore EUS-FNA should be performed only after the risks and benefits have been thoroughly evaluated.

\section{Competing interests: None}

\section{References}

1 Hong SK, Loren DE, Rogart JN et al. Targeted cyst wall puncture and aspiration during EUS-FNA increases the diagnostic yield of premalignant and malignant pancreatic cysts. Gastrointest Endosc 2012; 75: $775-782$

2 Tanaka M, Fernández-del Castillo C, Adsay Vet al. International consensus guidelines 2012 for the management of IPMN and MCN of the pancreas. Pancreatology 2012; 12: $183-197$

3 Pais SA, Attasaranya S, Leblanc JK et al. Role of endoscopic ultrasound in the diagnosis of intraductal papillary mucinous neoplasms: correlation with surgical histopathology. Clin Gastroenterol Hepatol 2007; 5: $489-495$ 
4 Ngamruengphong $S, X u C$, Woodward TA et al. Risk of gastric or peritoneal recurrence, and long-term outcomes, following pancreatic cancer resection with preoperative endosonographically guided fine needle aspiration. Endoscopy 2013; 45: 619-626

5 Levy MJ, Gleeson FC, Campion MB et al. Prospective cytological assessment of gastrointestinal luminal fluid acquired during EUS: a potential source of false-positive FNA and needle tract seeding. Am J Gastroenterol 2010; 105: $1311-1318$

6 Hirooka $Y$, Goto $H$, Itoh $A$ et al. Case of intraductal papillary mucinous tumor in which endosonography-guided fine-needle aspiration biopsy caused dissemination. J Gastroenterol Hepatol 2003; 18: $1323-$ 1324 